with drug-resistance, high transmissibility and clustering. But they do vary among endemic and sporadic strains, in different settings and hosts, even at within country level

Secondly, Russian epidemic clone Beijing B0/W148 was commonly regarded as widespread across all Former Soviet Union. In reality, its geographic distribution shows a peculiar clinal gradient with highest frequency in Siberia and sharp decrease in the Asian part of the former Soviet Union.

Thirdly, a global spread of LAM RD-Rio sublineage has been claimed and was attributed to its particular pathogenic properties. A comprehensive analysis of available data shows that RD-Rio strains are rarely present in Russia and East Asia. It appears that there is no global dissemination of RD-Rio due to specific virulence properties of these strains but rather their spread due to human migration (if such migration did take place).

A confusing terminology, misclassification and false clustering are not abstract issues but make a scientific discussion meaningless, and I will propose some courses for improvement of the situation.

Funding: Russian Science Foundation grant 14-14-00292.

6.34

doi: 10.15789/2220-7619-2018-4-6.34

\section{ANALYSIS OF SECONDARY RESISTANCE OF MYCOBACTERIUM TUBERCULOSIS TO SECOND-LINE ANTI-TUBERCULOSIS DRUGS IN CASABLANCA}

G. Momen $^{1,2}$, A. Aainoussi ${ }^{2}$, A. Lamaammal ${ }^{2}$, F. Chtioui ${ }^{2}$, M. Messaoudi' ${ }^{2}$, M. Blaghen ${ }^{1}$, M.D. El Messaoudi ${ }^{2}$, M. Khyatti ${ }^{2}$

${ }^{1}$ Faculté des Sciences Ain Chok, Casablanca, Morocco; ${ }^{2}$ Institut Pasteur du Maroc, Casablanca, Morocco

Tuberculosis (TB) is a major public health problem in Morocco, despite multiple strategies and funds allocated. According to epidemiological data, with about 31.452 TB cases detected in 2016, and a national incidence of about 91 per 100000 inhabitants per year, TB pose a serious threat to TB management in Morocco, with Casablanca being one of the most affected region.

The objective of this retrospective study was to evaluate secondary resistance of Mycobacterium tuberculosis to $2^{\text {nd }}$ line anti-tuberculosis drug in Casablanca.

In this retrospective study, 1300 patient samples from different CDTMRs and hospitals across Casablanca and regions over a 2 -year period from January $1^{\text {st }}, 2015$ to December 31 ${ }^{\text {st }}, 2016$ were analysed. Conventional techniques, such as BKD microscopic examination, BKC culture and antibiotic sensitivity were used for the diagnosis of TB.

Our results show that among the 1300 samples analyzed, $600(46 \%)$ were found positive for MTB, of which $58.33 \%$ were male and $41.66 \%$ were female. Patients aged between 20 and 40 years was the most affected group, representing $78 \%$ of patients. Data using the conventional Petroff decontamination and homogenization technique for isolation, identification as well as titration of the "BK" strains were as follows: Negative culture (54\%), Positive culture $(46 \%)$. The antibiogram used for this study gave the following results: $53 \%$ were wild strains, $47 \%$ were mutants, among which $18 \%$ were "MDR" strains and $1 \%$ were "XDR" strains.
The results of the present study reflect the importance of a good management of TB cases in order to succeed the treatment regime adopted at the national level and the success of the fight against this scourge in Morocco.

\subsection{5 doi: 10.15789/2220-7619-2018-4-6.35 DEVELOPMENT OF THE EXTERNAL QUALITY ASSESSMENT SCHEME FOR NON-TUBERCULOUS MYCOBACTERIA DRUG SUSCEPTIBILITY TESTING IN EUROPEAN UNION}

V. Nikolayevskyy ${ }^{1}$, F. Maurer ${ }^{2}$, Y. Holicka ${ }^{1}$, L. Taylor ${ }^{1}$, H. Liddy' ${ }^{1}$, D. Hillemann², ERLTB-Net network, K. Kranzer ${ }^{2}$

${ }^{1}$ Imperial College, London, United Kingdom; ${ }^{2}$ Nationales Referenzzentrum für Mykobakterien, Borstel, Germany

Non-tuberculosis mycobacteria (NTM) are increasingly associated with pulmonary and extrapulmonary disease in humans. Although identification and drug susceptibility testing (DST) of NTMs comprises a significant part of the tuberculosis (TB) reference laboratory activities in the European Union (EU), currently no internationally recognised external quality assurance (EQA) schemes exist for NTM DST. It lacks standardization and evidence on how to interpret DST results for specific drugs is limited.

Recognising the need for harmonization of methodologies in EU/EEA, European Reference Laboratory Network for Tuberculosis (ERLTB-Net) in 2017 conducted a pilot study among National Reference Laboratories (NRL) aimed at understanding methods employed for identification and DST and developing an EQA scheme for NTM DST. Pilot study comprised a survey followed by an EQA round using identical panels comprising 10 well characterised rapid (M. abscessus) and slow (M. avium) isolates.

Completed questionnaires were received from a total of 32 NRLs (97.0\% response rate). Thirty NRLs routinely perform identification of NTMs with a majority $(77.4 \% ; \mathrm{N}=24)$ using line probe assays as a primary means of NTM speciation. Reports containing minimum inhibitory concentrations (MICs) and result interpretation for five key drugs (Clarithromycin (CLA) Amikacin (AMK) Moxifloxacin (MOX); Linezolid (LIN), and Doxycycline (DOX) for $M$. abscessus only) were received from 21 NRLs (95.5\% response rate).

Interlaboratory agreement rates were higher for $M . a b$ scessus isolates; all five strains were found to be resistant to DOX by all NRLs (MIC $8.0-16.0 \mu \mathrm{g} / \mathrm{ml}$ ). Relatively minor variations were seen in MICs and their interpretations for MOX and AMK while for LIN MIC ranges for individual isolates varied greatly $(2.0-32.0 \mu \mathrm{g} / \mathrm{ml})$ across NRLs resulting in a lower agreement with regards to results interpretation. For slow growers AMK and MOX appeared to be the most problematic drugs both in terms of MIC determination (ranging 4.0-64.0 and 0.5-8 $\mu \mathrm{g}$ / $\mathrm{ml}$ in individual strains, respectively) and interpretation.

The results show that inter-laboratory reproducibility is insufficient, highlighting the need for expanding EQA schemes for NTM DST. As EQAs for M. tuberculosis complex DST have led to more reliable and reproducible phenotypic DST, we propose to follow a similar approach for clinically relevant NTM. 